the arrhythmia. In three cases recently reported by Dr. Chin and associates $^{3}$ there was good evidence, both on detailed electrocardiographic analysis and on the response to vagal stimulation, that the rhythm was nodal in origin.

Though the mortality rate of this arrhythmia is between $80 \%$ and $90 \%, 4$ Chin and colleagues suggest that the prognosis is not necessarily serious when an operation or an anaesthetic precipitates the arrhythmia or when it is associated with a reversible condition like hypokalaemia. They report on five patients, of whom only two died in the arrhythmia, a third dying later in renal failure. Two patients were eventually discharged from hospital. One, who was suffering from mitral stenosis, developed the arrhythmia during the anaesthetic for a second mitral valvotomy. The other, who was hypertensive and admitted to hospital with a stroke, was found to be hypokalaemic, with a serum potassium level of only $2.25 \mathrm{mEq} / 1$. Compression of the eyeballs in this case slowed the bidirectional tachycardia, and after $2 \mathrm{~g}$. of potassium had been given in an intravenous glucose drip sinus rhythm eventually returned.

While, therefore, bidirectional tachycardia is always potentially serious, it does not necessarily carry a bad prognosis when there is an associated precipitating cause and when the myocardium is not too severely damaged by the underlying cardiac lesion. Vagal stimulation and medicinal therapy, notably with potassium and magnesium, have in suitable cases been effective in arresting the arrhythmia. It is possible that, with the introduction of continuous electrocardiographic monitoring and of cardioversion, further progress will in the future be made in the recognition, analysis, and treatment of this condition.

\section{Phlegmasia Cerulea Dolens}

The syndrome of massive venous occlusion in a limb, with intense violaceous cyanosis, woody oedema, ecchymoses, loss of arterial pulsations, and sometimes gangrene, was called phlegmasia cerulea dolens (phlébite bleue) by $R$. Grégoire ${ }^{1}$ in 1938 to distinguish it from the commoner type of deep venous thrombosis known as phlegmasia alba dolens (white leg).

Phlegmasia cerulea dolens is rare; the number of reported cases is less than $100 .^{2}$ It is most often seen in association with malignant disease, but it can also occur in patients with pelvic infection and after operation or labour. In a few instances it has arisen as a primary condition. Frequently the main thrombosis is preceded by an apparently minor attack of thrombophlebitis, but there may be no such warning. The onset is sudden and dramatic, and pain is severe. In addition to the local symptoms the patient often suffers from hypovolaemic shock. The mortality is $30-40 \% .^{2}$ s

The syndrome is apparently due to an almost total occlusion of the venous drainage of the limb. Why this should occur is uncertain, but reports that after the occlusion the patient's blood may be incoagulable ${ }^{45}$ suggest that some biochemical event in the limb sets in train an explosive local reaction in which the clotting factors are rapidly used up. The disappearance of the peripheral pulses and the development of gangrene have been attributed to secondary arterial spasm, because in fatal cases it has regularly been found that there is no occlusion of the main arteries. It is more likely, however, that these events are due entirely to the massive venous occlusion causing a rise in pressure within the limb sufficient to impede the arterial inflow.

Various forms of therapy have been suggested. Whatever treatment is given to the limb restoration of the blood volume is often the first necessity. Some writers recommend raising the limb and the application of compression bandages and active and passive movements, ${ }^{6}$ but such measures may increase the risk of pulmonary embolism, seen in $40 \%$ of cases. 'J. M. Stallworth and his colleagues" state: "The importance of heparin and to a lesser degree vasodilators is well established." Yet there are good reasons for questioning the value of both these treatments. Phlegmasia cerulea dolens may develop in patients who are receiving apparently adequate anticoagulant therapy, and there is some evidence that anticoagulants may increase the amount of bleeding into the subcutaneous tissues and thus make the situation worse. ${ }^{3}$. Interruption of the sympathetic supply to the limb by procaine block, by ganglion-blocking drugs, or by operation, and the use of vasodilator drugs have been advocated by those who believe that arterial spasm is a factor in the condition, ${ }^{7}$ but the evidence that such measures are helpful is unconvincing, and it is surely illogical to try to get more blood into the limb so long as the outflow remains completely obstructed. Decompression fasciotomy is a more rational procedure and has been used with some success. ${ }^{3} 8$

Recently several reports have indicated that surgical removal of the thrombus is the best method of treatment. $\mathrm{H}$. Mahorner, ${ }^{9}$ writing of 16 years' experience of thrombectomy for major venous thrombosis, has no doubt that it is the method of choice. He opens the femoral vein and expresses the clot by pressure on the abdomen and by milking it upwards from the calf. Heparin is given postoperatively, starting 24 hours later, and he does not advocate proximal ligation of the inferior vena cava. T. J. Fogarty and his colleagues" treated six patients by thrombectomy and proximal ligation, the thrombus being removed by a Fogarty embolectomy catheter. All but one of the cases had a successful outcome. S. K. Brockman and J. S. Vasco ${ }^{10}$ reported two successful cases; in one of them the thrombectomy was combined with plication of the vena cava. N. L. Robles and others ${ }^{11}$ have also obtained good results from thrombectomy in three cases of phlegmasia cerulea dolens; they gave heparin after operation, beginning as early as five hours after the operation

Thrombectomy is a logical method of treatment, and these encouraging results suggest that it should be used more often. It is clearly difficult to be certain that all the thrombus has been removed, and there is always a danger of further thrombosis after the operation. To prevent purmonary embolism it is therefore necessary to ligate or to plicate the inferior vena cava at the time of operation or else to follow it with anticoagulant therapy. Both of these methods have their advocates, and at present it is not clear whether one is better than the other.

1 Grégoire, R., Presse méd., 1938, 46, 1313.

- Stallworth, J. M., Bradham, G. B., Kletke, R. R., and Price, R. G., Ann. Surg., 1965, 161, 802.

Fogarty, T. J., Cranley, J. J., Krause, R. J., Strasser, E. S., and Hafner, C. D., Arch. Surg., 1963, 86, 256.

4 Rosenberg, N., and Zullo, R. J., ibid., 1958, 76, 981.

s Meek, J. R., and Maurer, J. J., Amer. F. Surg., 1959, 97, 104.

Catchpole, B. N., Lancet, 1957, 1, 343.

Boyd, D. P., and Clarke, F. M., Surgery, 1962, 51, 19.

Cywes, S., and Louw, J. H., ibid., 1962, 51, 169.

- Mahorner, H., Proc. Mayo Clin., 1965, 40, 809.

10 Mrockman, S. K., and Vasco, J. S., Amer. F. Surg., 1965, 109, 485

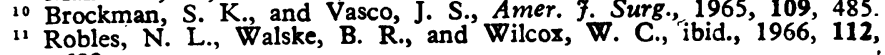
693. 